\title{
Retraction Note to: FoxM1 down-regulation leads to inhibition of proliferation, migration and invasion of breast cancer cells through the modulation of extra-cellular matrix degrading factors
}

Aamir Ahmad ${ }^{1}$ Zhiwei Wang ${ }^{1} \cdot$ Dejuan Kong ${ }^{1} \cdot$ Shadan $_{\text {Ali }}{ }^{1} \cdot$ Yiwei $_{\text {Li }}{ }^{1}$

Sanjeev Banerjee ${ }^{1} \cdot$ Raza Ali $^{1} \cdot$ Fazlul H. Sarkar ${ }^{1,2}$

Published online: 13 July 2016

(C) Springer Science+Business Media New York 2016

Retraction note to: Breast Cancer Res Treat (2010)

\section{2:337-346}

DOI 10.1007/s10549-009-0572-1

The paper is retracted at the request of the publisher because in Figs. 5a and 5b, and $6 \mathrm{c}$ micrograph images are re-used and re-labeled to represent different conditions.

The online version of the original article can be found under doi:10.1007/s10549-009-0572-1.

Fazlul H. Sarkar

fsarkar@med.wayne.edu

1 Department of Pathology, Barbara Ann Karmanos Cancer Center, Wayne State University School of Medicine, Detroit, MI 48201, USA

2 Department of Pathology, Karmanos Cancer Institute, 740 HWCRC Bldg, 4160 John R. Street, Detroit, MI 48201, USA 